$\left[{ }^{3} \mathrm{H}\right]$ thymidine incorporation 625

$15 \mathrm{~d}-\mathrm{PGJ} \mathrm{H}_{2} \quad 1303$

$16 \mathrm{q} 1763$

$17 \beta$-oestradiol 136

1q 1763

2-(4-aminophenyl) benzothiazole 1348

5-aminolaevulinic acid 989

5-aza-2'-deoxycytidine 979

5-fluoropyrimidine 1670

5-fluorouracil 1028, 1162, 1677,1871

5-FU 222

9-aminocamptothecin 1634

$\alpha$-foetoprotein 728

$\alpha$-particle emitter ${ }^{213} \mathrm{Bi} \quad 1197$

$\alpha_{\mathrm{v}}$-integrins 788

accelerated hyperfractionation 517

accelerated radiotherapy 1848

actin cytoskeleton 1188

actinomycin D 1174

active specific immunotherapy

(ASI) 1230

acute lymphoblastic leukaemia 1727

acute myeloid leukaemia 60 , 1061

adaptation 1540

adenocarcinoma 1238

adenocarcinoma of unknown primary 1884

adenocortical tumours 1561

adenovirus 436

adhesion 1169

adjuvant androgen deprivation 1843

adjuvant chemotherapy 1578

adjuvant treatment 1534

adolescent weight 84

adrenal cortex 1561

adult body size 84

advanced epithelial ovarian cancer 1385

advanced oesophageal cancer 669

adverse drug reaction 12

aetiology 60, 350, 1061, 1070, 1745

AF15Q14 1757

affected and unaffected women 43

agarose 947

age 1061,1373

Akt 540,1586

ALA 837

ALA derivatives 837

alcohol 346

ALL 356

alternating therapy 692

alternative splicing 737

AMF 1914 aminolaevulinic acid 1343 aminolevulinic acid 837

anaemia 1243

anaplastic astrocytoma 501

anaplastic thyroid carcinoma 1848

androgen receptor 879

androgens 879

angiogenesis 123, 244, 301, $389,417,490,558,744$,

761, 779, 788, 796, 864, 905, 993, 1391, 1465,

$1566,1597,1622,1803$, 1813

angiogenic factors 768,1276

angiopoietin 645

anthracycline-resistant 1367

anthracyclines 226, 811

anthranoids 1494

anti-angiogenesis $\quad 803,1813$

antiangiogenesis 788

anticancer drugs 1316

antidepressive agents 92

antioxidant activity 1645

antisense 1328, 1957

antivascular 1937

Apaf-1 587

Apaf-3 596

Aplidine 1510

apoptosis 130, 143, 443, 463 $587,636,737,744,828$, 893, 963, 971, 1012, 1104, $1188,1303,1316,1479$, $1494,1510,1776,1937$, 1951, 1957, 1963

aspirin 1501

ATP 619

autoantibodies 1824

auto-immune disease 1078

autologous melanoma vaccine 1534

autologous tumour cell

vaccines 1230

avidin-biotin 207

axillary node status 870

$\beta$-catenin 1124

$\beta$-glucuronidase 811

$\beta 3$-adenoceptor 424

$\beta$-carotene 698

B cells 1336

B lymphocytes 1776

B16 F10 melanoma 803

basic fibroblast growth factor $31,123,1770$

Bax 1117

BAX 744

Bayesian 843

Bcl-1 143, 971, 1117, 1501, 1561

BCL-2 886

bcl-2 1899

Bcl-2 phosphorylation 463

BCR-ABL 1472 benign thyroid disease 1745 bereavement 1540

beta 2-microglobulin 1943

Betaig-h3 1923

bile acids 1741

birth length 89

birth weight $84,89,728$

bisphosphonates 1479

bladder cancer 1283, 1732

bladder carcinoma 326

bladder neoplasms 1276

bleomycin 1658

body surface area 1297

bombesin receptor 1322

bone marrow micrometastasis 1047

bone marrow stroma 1136

bone mestastases 322, 1715

brain cancer 1732

brain tumour 14,1414

brain tumours 1070

brain-specific angiogenesis inhibitor 1490

BRCA1 mutation 218

BRCA2 mutation 218

BRCA3 76

breast 250, 905

breast and ovarian cancer genetics 43

breast cancer $84,89,218,222$, $362,540,580,723,728$, 750, 870, 1041, 1367, 1373, $1436,1440,1457,1479$, 1705, 1786

breast cancer predisposition 718

breast cancer prognosis 1790

breast cancer risk 718

breast carcinoma 546

breast feeding 1064

breast neoplasm 1034

breast neoplasms 92

breast tumour 136

butyrate transport 1262

CA $15-3 \quad 1217$

CA19-9 1551

cachexia 372, 424, 1628

Caco2 1645

calcium signalling 636

caloric restriction 1615

cancer 51, 636, 698, 1243, $1336,1634,1854,1963$

cancer cachexia 612, 1012

cancer chemotherapy 811,983

cancer in spouses 1737

cancer metastasis 161

cancer network 313

cancer testis antigens 979

cancer/testis (CT) 1757

cancer therapy 130, 449, 652

capecitabine 1367,1670

carbonic acid 1276

carboplatin 1379, 1385 carcinogenesis 1124, 1494

carcinoma 893, 1444

carcinoma of breast 396

carcinoma of unknown

primary site 1238

cardiolipin peroxidation

1951

cardiotoxicity 226, 534, 1697

cardiovascular disease 698

carnitine 1854

case-control $350,1721,1751$

case-control studies 92, 1425, 1085

case-control study 346,712 , 1436, 1745

case-mix 331

caspase 443

caspase activity 971

caspase-9 587

caspase-independent 828

caspases $143,893,1479$

caveolin-1 912

C-cell hyperplasia 1929

CCND1 expression 580

cdc25A 1909

cdc25B 1909

CD1a 546

CD20 1336

CD83 546

CEA 1217

cell adhesion 161

cell cycle 443, 1501, 1510, 1645

cell cycle markers 402

cell hypoxia 1276

cell line 98

cell surface molecules 1776

cell proliferating activity 1893

cellular proliferation 1776

cellular telephones 348

census 350

central nervous system

tumours 1727

central primitive neuro-

ectodermal tumours 1117

c-erbB-2 568

cervical cancer 263,1188

cervical carcinoma 705

cervical disease 269

CGH 929

change detection analysis 870

chemoembolization 196

chemo-immunotherapy 1230

chemoprevention 218, 774

chemoradiotherapy 552, 1551

chemotaxis 993

chemotherapy $19,26,179$,

213, 336, 485, 501, 669,

$744,1546,1555,1670$,

$1677,1684,1854,1905$

chemotherapy duration 1705

childhood 356

childhood/adolescence 362

childhood cancer 63, 350, 1078,1727 
childhood cancer risks 1721

childhood leukaemia 732 ,

$1064,1411,1419$

children 1070

CHK2 756

cholecystectomy 1081

chondrosarcoma 1303

choroid plexus 1592

chromatin 1817

chromosomal abnormalities 60,1061

chromosome 121763

chronic myelogenous

leukaemia 1487

cimetidine 161, 630, 1257

CIN 376

ciprofloxacin 443

circadian 999

circulating tumour cells 110

cisplatin 19, 196, 213, 326, $342,485,530,669,819$

cisplatin 1858

c-kit (CD117) 1333

clodronate 1235

cluster analysis 1093

CMRF-44 546

CMRF-56 546

coagulation 389

cohort studies 70, 1430

cohort study 723

colon 1494

colon cancer $1230,1262,1501$

colon carcinoma 409, 1871

Colon 38 tumour 1937

colonic cells 1316

colony stimulating factors 1316

colorectal 102

colorectal cancer 161, 203, 331, $443,568,574,417,1124$,

$1257,1401,1670,1684$

$1691,1741,1876,1884$

combination 534

combination therapy 819

combined treatment modality 1848

combretastatin A-4 1604

communication 51

communication skills 1057

comparative genomic

hybridisation 924

computer 51

computer-assisted cytology 382

conformal radiotherapy 1843

Congou tea 346

cost and cost analyses 226

cost effectiveness analysis 1677

costs 1684

CPT-11 983

cryosurgery 1658

crystallization 196

CTLs 151

CXCL12 1250

CXCR4 1250

cyclin A 402

cyclin BI promoter 477

cyclin D1 402

cyclooxygenase-2 1150 cyclophosphamide 130, 534 1705

CYP1A1 1348

CYP1B1 774

CYP26A1 605

cytarabine 1472

cytochrome $c$ 893, 1479, 1951

cytochrome P450 630, 774

cytogenetics 60,1061

cytokeratin-positive cells 1047

cytokeratin staining 1884

cytokine 449

cytokines 1310

D40 1757

daycare 1064, 1419

DC activation 546

declopramide 971

delayed exposure 1419

delayed type hypersensitivity (DTH) 1534

dendritic cell 1257, 1529

dendritic cells 151, 546

deoxycholic acid 1741

deprivation 331

DFF 596

diabetes 1078

diagnosis 1444

diagnostic delay 1034

diet 712

dietary factors 1745

differentiation 117,1006

diffusion 1639

disease-progression 31

divalent-Fab' maleimide 1401

DMXAA 1937

DNA breaks 130

DNA double-stranded breaks 1143

DNA fragmentation 1012

DNA methylation 282, 574, 1817,1888

DNA mismatch repair 1130

DNA ploidy 1110

DNA repair 130,1797

DNA sequencing 1117

DNA-PK 1143

do not attempt resuscitation orders 1057

docetaxel 326, 692, 1385

dose calculation 1297

dose response 1235

dose-dense chemotherapy 1555

dose-finding study 1385

doxorubicin 1093, 1322, 1697, 1848

DPD 222, 1028

DPYD gene 1028

drug delivery 652

drug interactions 1162

drug resistance 1130, 1652

DS-sarcoma 1355

ductal carcinoma in situ 905

E-cadherin 98

E6 oncogene 269

early infections 1064

early life factors 356
EBERs 702

EGF receptor 456

EGFR 1518

EGFR-TKI 1157

endocrine treatment 540

endocytic vesicles 652

endogenous superantigen 828

endometrial cancer 1837

endometrial neoplasms 1430

endometrium 761

endothelial 1937

endothelial-cell 1597

endothelial cells $645,779,993$, 1813

endothelial phenotype 905

endothelial proliferation index 761

energy metabolism 424, 1615

energy restriction 362

ependymoma 929

epidemiology 92, 348, 698, 1411,1751

epidermal growth factor

receptor 1328

epirubicin 1385

epithelial ovarian cancer 879

Epstein-Barr virus 702

$\mathrm{ER} \beta \quad 250$

ErbB-2 540

ErbB2 1104

erythropoietin 1858

ethnicity 367

etoposide 12, 19, 485, 1472

etoposide phosphate 12

evidence-based medicine 313

Ewing tumours 1763

exercise 70

expression 917

expression cloning 1943

extracellular matrix 1639

Fab 954

familial breast cancer 233

familial cancer 718,1737

family 1540

Fas 963

fatigue 1854

FEC 692

female serum 203

FHIT 376

fibrinogen 389

fibroblast growth factor 81097

FIGO stages 274

first line therapy 1677

FISH 1449

flavonoids 1645

Fluoro-desoxi-glucose uptake 1391

fluoropyrimidine 1367

fluorouracil 819

fragments 1401

free PSA 203

frozen tissue 1658

functional genomics 1250

G0/G1 arrest 1150

gain Iq 929

galanin 117 gallstones 1081

gamma dose rate 1727

gastric adenocarcinoma 702

gastric cancer 564, 674, 1047 , $1578,1858,1864$

gastric carcinoma 1223

gelatinase activity assay 1876

gelatin zymography 1876

gemcitabine 190, 342, 843, 1551,1701

gene amplification 1449

gene expression 274, 1093, 1262

gene expression patterns 1283

gene overexpression 899

gene therapy 436

genetic epidemiology 1737

genetic risk assessment 233

germ cell cancer 506

germ-cell tumours 1555

Glasgow osteosarcoma 999

Gleevec 1487

glioblastoma 1328

glioblastoma mutliforme 501

glioma 14, 207, 587, 619, 1586,1615

glioma c6 1006

Glivec 1487

glucose transport 1262

glucuronide 811,1634

glutathione 1180

glycolysis 1615

glypican $3 \quad 1920$

GM-CSF 292

Gompertz equation 947

grade 185

graft- $v s$-leukaemia 828

growth control 1023

$\mathrm{H}_{2}$ receptor antagonist 1257

haem metabolism 630

haemoglobin 1243

hCG $\beta \quad 185$

hDFM 1401

head and neck cancer 512, 517

head and neck cancer cell lines 819

head and neck neoplasms 196

health behaviour 1034

heparanase 1270

heparin 1803

hepatic artery infusion 436

hepatobiliary cancer 1732

hepatocarcinogenesis 630

hepatocellular carcinoma 98 , 110,524

HER-2/neu 1449

heredity 1737

heregulin $\beta \quad 1540$

hexyl-ALA 837

HIF-1 1914

high-dose chemotherapy 506

high-grade glioma 501, 1899

high-risk families 76

histological grade 396

histopathological differentiation 1893

histone acetylation 1817 
historical cohort 356

HLA class I 1336

HMG Co-A reductase inhibitors 1436

hMLH1 574

hMLH1 564

Hodgkin and Reed Sternberg cells 1333

hormone refractory 1715

hospital 331

HPV 263, 376

human 1444

human breast cancer 402, 1097

human bronchial epithelial cells 1923

human fusion protein 811

human granulocyte colonystimulating factor 1858

human kallikrein $6 \quad 1790$

human kallikrein $10 \quad 1790$

human kallikrein $13 \quad 1457$

human papillomavirus (HPV) 382, 705, 924

human papillomavirus 16269

human squamous cervical cancer 274

humanised antibody 1401

hyaluronan 947

hypercalcaemia 680,1235

hyperlipidaemia 1436

hypermethylation 564

hypersensitivity 12

hyperthermia 1597

hypoxia 136, 301, 1355, 1914

hypoxia markers 1831

IAPs 737

ICAD 596

IL-1 $\beta \quad 1396$

IL-4 receptor 285

IL-6 1396

IL-8 301

image processing 1831

imaging 1401

imatinib 1487

immune activation 1691

immune infiltrate 1444

immune monitoring 168

immunofluorescence 285

immunohistochemistry 222,

$568,912,1449,1770,1831$, 1888,1909

immunotherapy 336, 979, 1336

incidence rate 1061

individual 51

induction chemotherapy 530

infection $732,1070,1414$

infiltrating lymphocytes 1465

inflammation 1615

infusional 5-FU 213

inhibition 768

inhibitor 605

inhibitor of apoptosis 886

insulin-like growth factor-

binding protein-3

(IGFBP-3) 1963

integrin receptor 1923

interferon $\alpha 179$ interleukin-2 179

interleukin-6 389

intermediate ploidy 929

internal ribosome entry sites (IRES) 1023

International Germ-Cell Cancer Consensus Group 1555

International Neuroblastoma Pathology Classification (INPC) 1110

International Neuroblastoma Staging System (INSS) 1110 interventional radiology 524 intradermal 336

intramuscular administration 63

intra-pleural 336

intratumour injection 429

intravenous infusions 1670

invasive carcinoma 905

Ip deletion 596, 1110

Iressa 456, 1157

irinotecan 530, 999, 1677

irradiation 1487

isolated limb perfusion 1174

isolated liver perfusion 436

in vitro cell model 1283

Jak 449

JNK 1188

kallikreins 1457

Ki-67 1561

kidney 768

kidney cancer 1250

kidney neoplasms 1425

Kuwait 1745

lactation 1097

laminin 1169

landfills 1732

larynx 239, 1751

late rectal toxicity 1843

leukaemia 63, 143, 292, 1414, 1721,1732

Li Fraumeni 1592

light dose fractionation 989

lipid mobilizing factor 424,612

liposomal doxorubicin 1379

liposomes 837

liquid-based cytology 382

liver cancer 625

liver cirrhosis 1290

liver metastases of colorectal cancer 524

liver metastasis 436

locally advanced NSCLC 190

locoregional chemotherapy 524

long control region 269

losoxantrone 534

loss 22929

loss of heterozygosity 574

LoVo xenograft 1157

LMR-12 1943

LRP 954

Lsm1 940

lung 1751 lung adenocarcinoma 1884

lung cancer 36, 244, 1449 , 1757,1893

lymphoma 1414

MAGE transcript 110

male genital tract 705

malignant pleural

mesothelioma 342

malignant progression 1355

MAPK 1518

marimastat 1864

mathematical model 14

matrix metalloproteinase 1136,1876

matrix metalloproteinase inhibitors 1864

MCF 1238

MDM2 1290

medical audit 313

medical practice 313

medulloblastoma 285

melanoma 102, 179, 301, $1085,1529,1957$

menopause 1430

mesothelioma 979

metabolism 605

metastasis 123, 301, 389, 409, 744, 803, 1136, 1169, 1604, 1803,1914

metastatic breast cancer 692, 1905

metastatic colorectal cancer 1677

metastatic gastric cancer 213

methotrexate and fluorouracil (CMF) 1705

methylating agents 1797

methylation 979

metoclopramide 971

MGMT 1797

MHC class I 1943

microarray 1093

microcirculation 1604

microenvironment 1615

micrometastases 396

microvessel count 1770

microvessel density 1391, 1566, 1905

migration 1169

minimal-disease 102

mitochondria 619,1951

Mitomycin C 470

MMACI 257

MMPs 993

MO7e 292

molecular cytogenetics 1763

molecular diagnosis marker 274

monoclonal 250

monocytes 1310

motility 1914

MRS 963

m-THPC 1130

multidrug resistance 292, 954, 1494, 1943

multidrug resistance-associated protein 11578 multiple cancer 564

multiple comparisons 1093

multiple gated acquisition scan 226

multiple myeloma 796

multivariate analysis 1217, 1899

muscle 372

mutation 1028, 1920

MVD 796

MVP 954

Mxi1 477

MYCN amplification 1110

myo-inositol 1180

myotubes 1628

N-(4-hydroxyphenyl)retinamide 1951

NASBA 102

nasopharyngeal carcinoma 843

natural compound 1510

NDGA 1188

neoadjuvant androgen

deprivation 686

neoadjuvant chemotherapy

190, 750, 1041

neoplasias 917

neoplasm 768, 924

neoplasm staging 1034

nested polymerase chain

reaction 110

neuroblastic tumour 117

neuroblastoma $117,485,596$, 1110

neuroectodermal 596

nipple aspirate fluid 1440

nitric oxide 1310

nitric oxide synthase 136

nitric oxide synthase II 1310

nitrosoureas 1797

NMR 1180

non-anticoagulant heparincarrying polystyrene

(NAC-HCPS) 1803

non-Hodgkin's lymphoma 1414, 1727, 1770

non-seminomatous germ-cell tumours 1555

non-small cell lung cancer 530, 1701

normal 1963

NS-398 1150, 1501

NSCLC 858

NUCB2 1824

nutrient deprivation 1343

$O^{6}$-methylguanine-DNA

methyltransferase 1888

occult metastases 396

occupational physical activity

70

ocular melanoma 348

oesophageal cancer 346, 552

oesophageal carcinoma 1223

oesophagectomy 851

oesophagus 1751

oestradiol 728

oestrogen receptor 250, 282 
oestrogens 367, 1430

omega-3 fatty acids 983

oncogene overexpression 1449

ONYX-015 5

OPS 1622

oral 1670,1751

oral cavity 239

oral contraceptives 1085

organometallic arene

complexes 1652

osteosarcoma 864

ovarian cancer 19, 282, 456,

$712,1180,1379$

ovarian surface epithelium 879

ovarian xenografts 1652

ovulation 367

oxaliplatin 999, 1162, 1871

oxidative stress 630,779

\section{P14ARF 1290}

p21 1150

p21 WAF1/CIP1 625

p53 5, 490, 587, 744, 750, 756,

886, 1104, 1518, 1561, 1592

p53 proteins 1117

p73 263

p9Ka 409

paclitaxel 669, 1367, 1379, 1858

palliative chemotherapy 1858

pancreas 257, 893

pancreatic cancer 680, 1081, 1188,1551

pancreatic ductal

adenocarcinoma 1270

pancreatic neoplasms 886

Pap smear 382

parathyroid hormone-related peptide 322

parity 1425

participation 350

paternal occupational contacts 732

pathological response 1041

pathology 674

PC3 cell line 1197

PCR - RFLP 568

p-dimethylaminoazobenzene 630

PDT 837

peptide 1169,1529

perforin 828

pericyte 645

periodate-treated 1803

peripheral blood 1715

peripheral blood stem cell support 1715

PET 506, 1391

P-glycoprotein 1578

phage display 954

pharmacogenetics 1028

pharynx 239

phase I study 1701

phase I/II trial 1401

Phase II clinical trial 843

phase II study 342 phosphorylated tyrosinecontaining proteins 1893

phosphorothioate oligonucleotides 1143

photochemical 652

photodynamic therapy 837, $989,1130,1343$

piceatannol 774

pimonidazole 429

PKB 540, 1586

placenta weight 89

plasminogen activator inhibitor type 21197

plasminogen activator inhibitor type-1 1355

pleiotrophin 858

Polygenes 76

polymorphism 568, 1929

polypoid tumour 1124

pooled analysis 1085

poor prognosis 1893

population mixing 1411

population studies 76

population study 1837

porphobilinogen deaminase 1006

positron emission tomography (PET) 512, 1052

postoperative radiotherapy 517

postoperative survival 1270

PPAR $\gamma 1303$

predictive factor 1905

pre-eclampsia 728

Pre-phase I 1052

predictive factors 517, 1705

predictive genetic testing 1209

pregnancy 1425

pregnancy choriocarcinoma 26

primary breast cancer 1217

probability mapping 870

prodrug 1348, 1634

prognosis $185,222,402,417$, $568,1047,1217,1578$

prognostic and predictive markers 1457

prognostic factor 1041

prognostic factors 674,686

prognostic indicators 1797

prognostic markers 1763

prognostic value 580

proliferation 443, 636, 1957 , 1963

proliferation kinetics 517

prospective cohort study 1081

prostate 102

prostate cancer $168,322,686$, $940,1136,1197,1715$

prostate carcinoma 1465, 1843

prostate epithelia 1136

prostate specific antigen 1465

prostatic neoplasms 70

proteolysis-inducing factor (PIF) 1628

proteomics 1440

protoporphyrin 1006

PSA 168, 203

PSA control

psychological 1540 psychological distress 43

psychological impact 233

PTEN 1586

pulmonary metastasis 864

purinergic receptor 636

quality of life $51,1243,1691$

quantitative PCR 1457

quantitative RT-PCR 580

R116010 605

RA 605

radiation 819, 1929

radiation response 136

radiation sensitivity 1143

radioimmunotherapy 207

radiolabelled anti-PEM 870

radiolabelled humanised

monoclonal antibody

imaging 870

radionuclide therapy 1715

radionuclide ventriculography 1697

radiopharmaceutical 1052

radiotherapy $470,686,744$, 1157

radon 1721

radon interactions 1727

raltitrexed 1871

Ras 1479

ras oncogene 436

rat aortic ring assay 796

rats 1174

reactive oxygen species 1951

real-time PCR detection 580

real-time RT-PCR 899

receiver operator characteristic

curve 899

receptor 117

receptor signalling 1250

rectal cancer 744

recurrence 1223

referral patterns 36

relapse 26

renal cell carcinoma 185,1396

reperfusion injury 989

replication-selective

adenoviruses 5

repopulation 470

reproducibility 1741

reproductive factors 723

residence 362

response monitoring 506

response to treatment 1790

resuscitation 1057

resveratrol 774

RET 1929

RET/PTC 1929

RGD-peptides 788

rhenium-186 HEDT 1715

ribosomal protein S12 274

risk factors 70, 712, 723, 1064, $1081,1373,1425$

risk management 1209

risk perceptions 43

RT-PCR 102, 737, 1322

ruthenium 993

ruthenium(II) 1652
S100A4 409

salvage regimen 1871

SCLC 858

Scotland 1837

seasonal variation 1070

secondary lung cancer 1391

secondary malignancy 239

segregation analysis 76

sequential therapy 692

SEREX 1824

serine proteases 1457

serous papillary uterine cancer 151

service delivery 233

sexual function 1546

sexual habits 705

sialyl Lewis antigens 161

side effects 1546

signal transduction pathway 449

SIR2 917

skeletal muscle 1012

small cell lung cancer 558

smoking 1430, 1757

smooth muscle 645

Snail 98

socio-demographic factors 1034

SOD-1 779

solid stress 947

solid tumours 1566

sorbitol 1180

space-time clustering 1070

spheroid 947

spheroids 1639

squamous cell cancer 239

squamous cell carcinoma 470 , 512, 924, 1150

SR 25989803

ST1571 1487

stage 185

Stage A chronic lymphocytic leukaemia 31

staging 1837

statins 1436

statistical methods 1093

STI571 1333, 1472

stomach neoplasms 1888

stromal cells 1639

structure activity relationships 1652

subunit composition 285

suramin 1143

surgery 1848

surgical treatment 1223

surveillance 239

survival $331,512,674,737$, 1790

survival analysis 1837, 1888

suspensions 196

Sweden 732

SYBR Green I 899

sympathetic nervous system 117

symptom control 1540

symptoms 1373

synergy 485

systematic review 501 
TACCI 1824

tamoxifen 218, 402, 761, 1546

tamoxifen therapy 1790

TAP 1943

targeted $\alpha$ therapy 1197

taxotere (docetaxel) 463

Tbdn-I 1824

Tc-99m hHMFG1 870

T-cell functions 168

tegafur 1670

telomerase 917, 1328

temozolomide 501

terminal care 1540

testis 1757

TGF- $\beta 1963$

TGF beta 257

theoretical model 14

thienopyridine 803

thiram 779

thrombosis 1813

thymidine phosphorylase 552, 1465

thymidylate synthase 1578

thyroid 917

thyroid cancer 1745

thyroid carcinoma 912

thyroid tumour 1909

thyroid tumours 1929

timing of surgery 1786

T-lymphocytes 409

TNF $\alpha$ 1012, 1396 tobacco 346

tobacco smoke 60

topoisomerase 11634

topoisomerase inhibitors 1501

toxicity 1634

toxicology 1052

transfection 1586

transformation 257, 1444

translation 1023

translation control 1957

translation initiation factor

(eIFs) 1023

treatment by specialist 36

treatment preference 851

treatment response 552

triacylglycerols 963

trophoblastic disease 26

tryptophan 1691

tumour 26, 257, 490, 645, 788

tumour antigens 1824

tumour-associated

macrophages 1465

tumour blood flow 1604

tumour cells 1310

tumour growth 1803, 1813

tumour hypoxia 429, 619

tumour infiltrating eosinophils 674

tumour lysate 151

tumour markers 506, 1217

tumour metastasis 1597 tumour microcirculation 1622

tumour necrosis factor $\alpha 1174$

tumour oxygenation 1831

tumour progression 940

tumours 1639

tumour size 396

tumour-specific marker 110

tumour suppression 1923

tumour suppressor 447

tumour suppressor gene 1586

tumour targeting 1322

tumour vascularity 123

tumour vessel 1604

tyrosine kinase 1472

tyrosine kinase inhibitor 456, 1518

ubiquitin-proteasome proteolysis 1628

UFT 1670, 1701

ulcerative tumour 1124

uncoupling proteins 372,612

under-dose 1297

urokinase plasminogen

activator $1136,1197,1355$

urothelial cancer 326

utilities 851

vaccination 168

vaccine 336,1529

vaccines 1336 vascular density 761

vascular endothelial growth

factor $31,389,552,744$

vascular hot spots 301

vascular phenotype 244

vasculature 645

VEGF 301, 417, 558, 858, 864, 1597, 1786

vinflunine 143

viral variant 269

virtual Northern blot 899

vitamin D 680

vitamin K 63

$\mathrm{v}-\mathrm{Src} 463$

vulva 924

vulval cancer 756

Waf-1 1117

waiting times 36

WiDr cell line 1162

Wilm's tumour 1920

woman 203

worry 1209

xenograft 470

yeast assay 750

ZD 1839 456, 819, 1157, 1518 